Article

\title{
Tobacco OPBP1 Enhances Salt Tolerance and Disease Resistance of Transgenic Rice
}

\section{Xujun Chen and Zejian Guo *}

Key Laboratory of Plant Pathology, Ministry of Agriculture; Department of Plant Pathology, China Agricultural University, Beijing 100193, China. E-Mail: chenxj@cau.edu.cn

* Author to whom correspondence should be addressed; E-Mail: guozj@cau.edu.cn; Tel. +86-10-62733849

Received: 19 November 2008; in revised form: 4 December 2008 / Accepted: 9 December 2008 / Published: 11 December 2008

\begin{abstract}
Osmotin promoter binding protein 1 (OPBP1), an AP2/ERF transcription factor of tobacco, has been demonstrated to function in disease resistance and salt tolerance in tobacco. To increase stress tolerant capability of rice, we generated rice plants with an $O P B P 1$ overexpressing construct. Salinity shock treatment with $250 \mathrm{mM}$ $\mathrm{NaCl}$ indicated that most of the OPBP1 transgenic plants can survive, whereas the control seedlings cannot. Similar recovery was found by using the seedlings grown in $200 \mathrm{mM} \mathrm{NaCl}$ for two weeks. The OPBP1 transgenic and control plants were also studied for oxidative stress tolerance by treatment with paraquat, showing the transgenic lines were damaged less in comparison with the control plants. Further, the OPBP1 overexpression lines exhibited enhanced resistance to infections of Magnaporthe oryzae and Rhizoctonia solani pathogens. Gene expressing analysis showed increase in mRNA accumulation of several stress related genes. These results suggest that expression of $O P B P 1$ gene increase the detoxification capability of rice.
\end{abstract}

Keywords: AP2/EREBP, salt tolerance, disease resistance, transcription factor, transgenic rice. 


\section{Introduction}

As sessile organisms, plants have developed adaptive strategies to cope with environmental stress by expressing specific genes and synthesizing a variety of proteins or compounds including transcription factors, late embryogenesis-abundant (LEA) proteins, transporters, antioxidants, proline, and sugars [1]. They are categorized either as effectors, which directly modulate or attenuate stress effects, or as regulators that are involved in stress perception, signal transduction, or regulation of effectors. LEA proteins have been considered to play roles in maintenance membrane structures, binding of water, and acting as molecular chaperones [2]. Sugar and other compatible solutes serve as osmoprotectants and stabilize biomolecules. For instance, accumulation of trehalose, a nonreducing disaccharide of glucose, in rice plants confers high tolerance levels to different abiotic stresses [3].

AP2/ERFs have been identified as a large gene family of plant transcription factors and contain a conserved basic 58 or 59-amino acid DNA-binding domain, defined as ERF domain [4, 5]. A comprehensive computational analysis identified 122 and 139 ERF family genes in Arabidopsis and rice, respectively [6]. ERF proteins have found to play important roles in plant adaptation to abiotic stress, such as drought, cold, salt oxidative stress and ABA response [7]. The barley ERF protein HvRAF enhances salt tolerance in Arabidopsis [8]. Three Arabidopsis ERFs, DREB1A, DREB2A, and $\mathrm{CBF} 1$, are induced in response to drought or cold stress [5,9]. Further, ectopic expression of the genes such as CBF1 or DREB1A has been shown to improve dehydration stress tolerance in Arabidopsis [10]. Dubouzet et al. [11] isolated five DREB homologs from rice, in which overexpression OsDREB1A increased tolerant to drought in Arabidopsis. In previous study, we have demonstrated that OPBP1, an ERF transcription factor isolated from tobacco is mediated in disease resistance and salt tolerance in tobacco [12]. In this report, we generated $O P B P 1$ overexpressing rice plants and found the transgenic progenies exhibited enhanced disease resistance against Magnaporthe oryzae and Rhizoctonia solani, as well as tolerance to salt stress.

\section{Results}

\subsection{Expression of stress-inducible genes in OPBP1 transgenic plants}

Since OPBP1 was a regulator of disease resistance and salt tolerance in tobacco, we extended the study to examine a possible biological function of OPBP1 in rice. OPBP1 gene was constructed under the control of a maize ubiquitin promoter (Ubi:OPBP1). Thirty independent lines were obtained by Agrobacterium-mediated transformation method. Expression of the transgene in some of $\mathrm{T}_{2}$ progenies was examined by northern blot analysis. As shown in Figure 1A, accumulation of OPBP1 mRNA was increased in roots, although the level of accumulation varied between different lines.

To examine the induction of gene expression in OPBP1 overexpressing plants, we chose several stress-inducible rice genes, such as delta-1-pyrroline-5-carboxylate synthetase (OsP5CS) [13], OsGSTu1 [14] encoding for glutathione-S-transferase, and OsIM1 [15], sharing homology with AOX members of AtIM and PTOX. Overall, expression of OsGST, OsIM1 and OsP5CS genes was increased in the transgenic $O P B P 1$ plants in comparison with the control plants (Figure 1B). 
Figure 1. Constitutively expressing $O P B P 1$ and stress-inducible genes in transgenic rice plants.

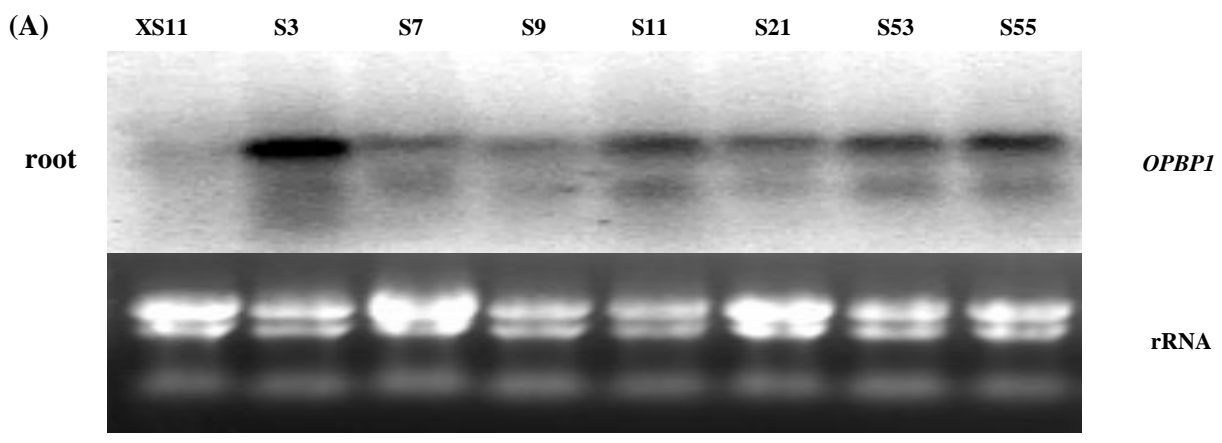

(B)

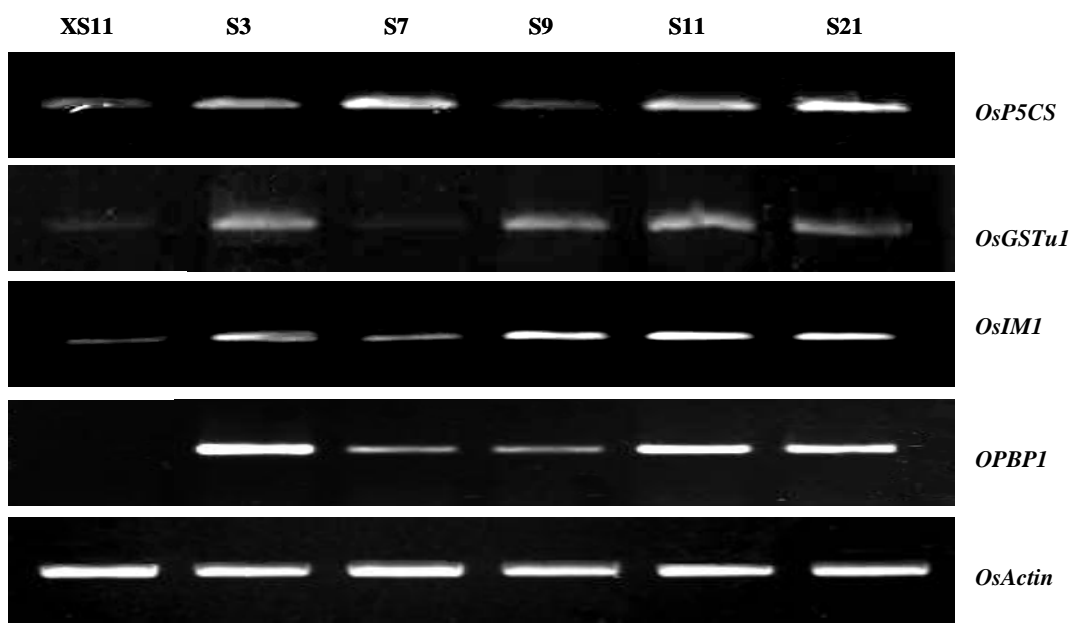

(A) Expression of OPBP1 gene in $C o U:: O P B P 1$ transgenic plants and in a control plant under normal condition was examined by Northern blot analysis using OPBP1 cDNA as a probe. Numbers indicate independent lines of transgenic $\mathrm{T}_{2}$ plants. (B) Expression of OPBP1, OsP5CS, OsGSTu1, OsIM1, and OsActin were determined by RT-PCR. The mRNAs from control plants (XS11) and CoU::OPBP1 lines were reverse transcribed and then used as templates for PCR. Actin gene was used as an internal standard. The amplification cycles were 25. Numbers indicate independent lines of transgenic $\mathrm{T}_{2}$ plants, and XS11 is the control.

\subsection{Expression of OPBP1 enhances salt tolerance in rice}

To investigate the response to salinity, the transgenic rice seedlings of $\mathrm{T}_{2}$ progenies were grown for two weeks in $1 / 2 \mathrm{MS}$ containing different concentrations of $\mathrm{NaCl}$ in the presence of hygromycin. The growth was suppressed with the increase of $\mathrm{NaCl}$ concentration both in the transgenic and the control plants. The heights in the presence of $200 \mathrm{mM} \mathrm{NaCl}$ were about one quarter of those without $\mathrm{NaCl}$ for both the control and transgenic lines (data not shown). Comparing the root growth between the transgenic and control plants, the length of roots in $O P B P 1$ overexpressing plants was even slightly increased in the presence of 100 or $150 \mathrm{mM} \mathrm{NaCl}$, whereas the root length did not change significantly in the control plants under this condition (Figure 2). Increase of $\mathrm{NaCl}$ up to $200 \mathrm{mM}$, the root growth 
was inhibited in both the transgenic and control lines. The effects of salt on plants are considered to be caused by ionic toxicity and osmotic pressure [16]. To examine the osmotic effect, rice seedlings were treated with osmotic reagent PEG $6000(12.1 \%$, w/v), whose osmotic pressure was about that of 100 $\mathrm{mM} \mathrm{NaCl}$. At this concentration of PEG6000, the root growth was even stimulated slightly at the osmotic stress (Figure 2C). The results suggest that inhibition of rice growth by $\mathrm{NaCl}$ treatment is possibly due to the ionic toxicity.

Figure 2. Effect of salt stress on rice seedlings of control and transgenic lines.

(A)

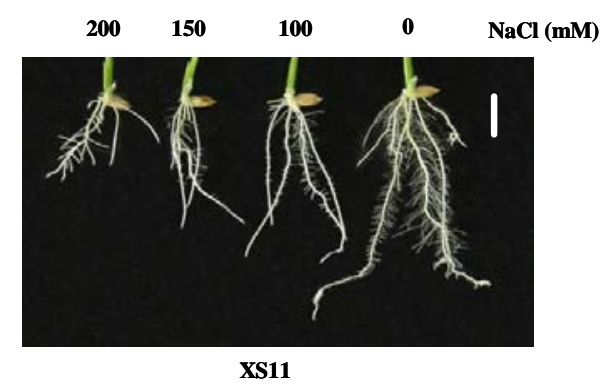

(B)

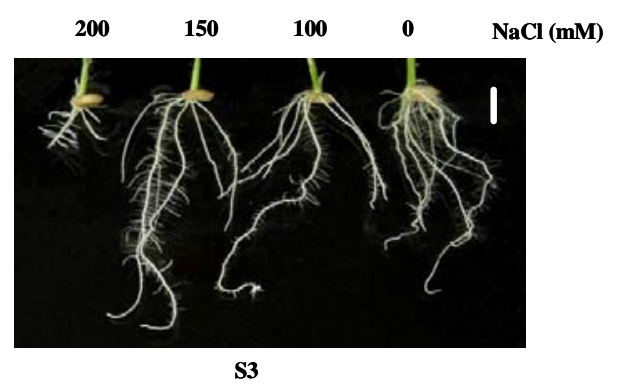

(C)

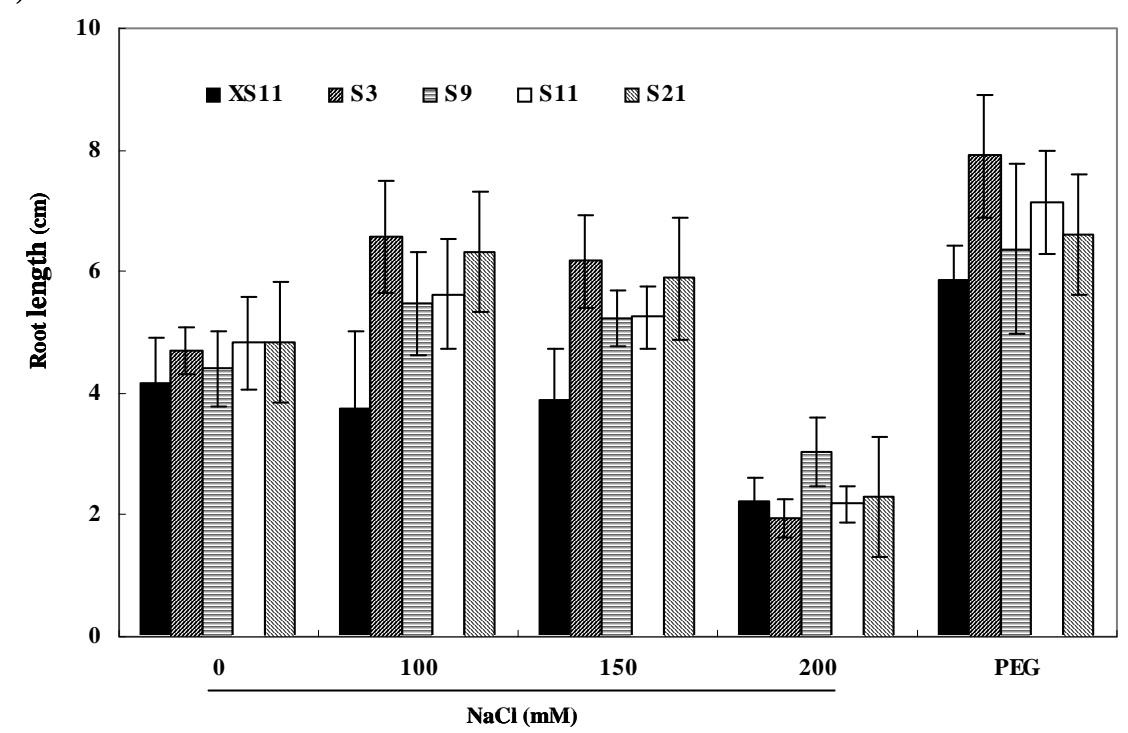

The germinated seeds were planted onto $1 / 2 \mathrm{MS}$ with $200,150,100 \mathrm{mM}$ or without $\mathrm{NaCl}$, respectively. Photos were taken after 14 days of growth. (A) The root growth of control plant (XS11) under salt stress. (B) The root growth of overexpression line (S3) under salt stress. The bar indicates $2.0 \mathrm{~cm}$. (C) The root length of each line under the same treatment. Values are means of at least 10 different plants in each line detected, and bars are standard errors. Numbers indicate independent lines of transgenic $\mathrm{T}_{2}$ plants, and XS11 is a control one.

To examine the recovery capacity of salt-treated plants, the rice seedlings grown in $200 \mathrm{mM} \mathrm{NaCl}$ for two weeks were transferred to normal 1/2MS medium for recovery. After another two weeks, the control plants did not grow significantly, as revealed by measurement of the plant heights (Figure 3A). 
Figure 3. The recovery growth of rice plant after salt stress.

(A)

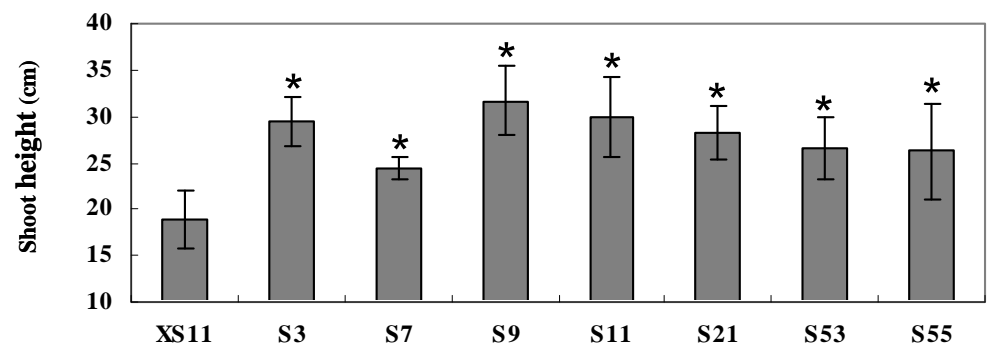

(B)

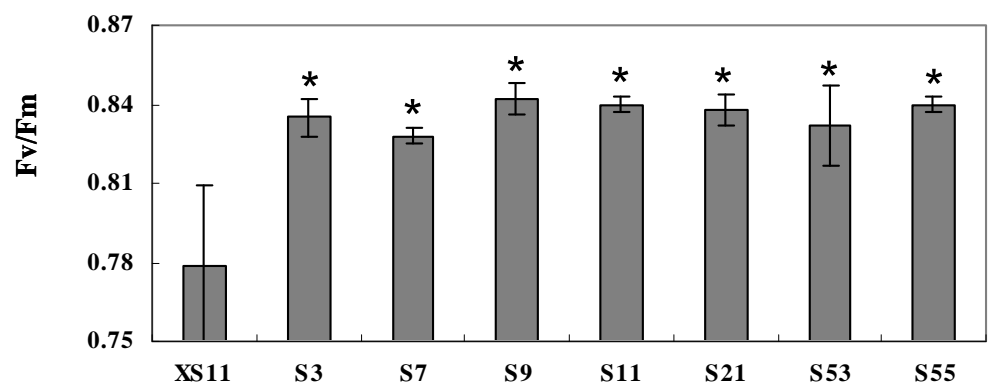

(C)

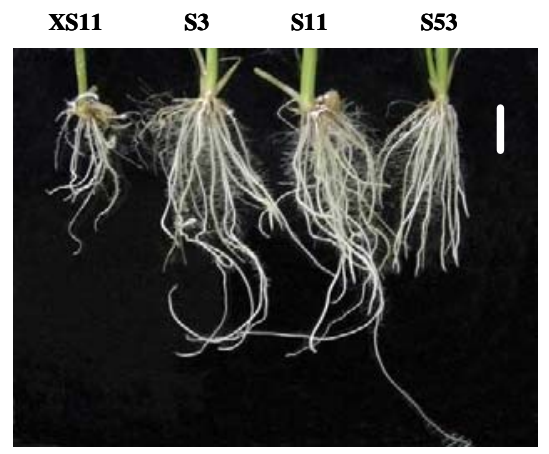

(D)

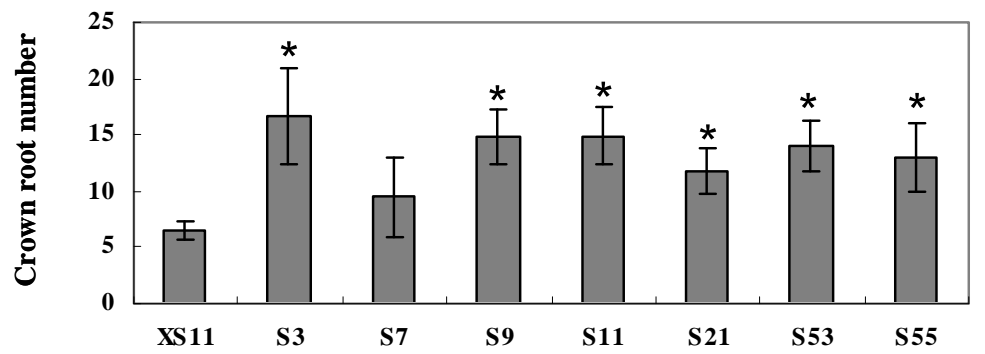

The seedlings from the $1 / 2 \mathrm{MS}$ medium containing $200 \mathrm{mM} \mathrm{NaCl}$ were transferred to normal 1/2MS medium for another 14-day recovery. (A) The shoot height was measured. (B) The second leaf was taken to measure Fv/Fm. Values are means of at least 10 different plants in each line detected, and bars are standard errors. The values of the overexpressing lines differ significantly with the control's $(\mathrm{P}<0.05)$ according to Fisher's protected LSD means comparison procedures. $(\mathrm{C})$ The root growth, the bar indicates $1.0 \mathrm{~cm}$. (D) The crown root number was counted after 14-day recovery. Numbers indicate independent lines of transgenic $\mathrm{T}_{2}$ plants, and XS11 is a control. The values of the overexpressing lines except $\mathrm{S} 7$ differ significantly with the control's $(\mathrm{P}<0.05)$ according to Fisher's protected LSD means comparison procedures. 
In line with this, the Fv/Fm value of the control plants, a parameter often used as an indicator of the efficiency of primary photochemistry in photosystem II (PSII), decreased in comparing with the $O P B P 1$ expressing lines (Figure 3B). Importantly, the number of crown roots was more in the $U b i: O P B P 1$ plants than the controls and the lengths of crown roots were longer (Figure 3C, D). Also, for most of the OPBP1 transgenic plants, the average biomass of the seedlings were near double the weight of the controls (data not shown).

Furthermore, the OPBP1 transgenic and control plants were examined for their restorable capability after high salinity shock. Three-week-old rice seedlings were exposed to $250 \mathrm{mM} \mathrm{NaCl}$ for $18 \mathrm{~h}$. With this treatment, about $37 \%$ of the youngest leaves of the control seedlings showed morphological wilting. Then, the treated seedlings were washed off and planted in soil for restoration. Within a seven-day-period of recovery, the control seedlings did not show obvious growth and about $31 \%$ of them died (Figure 4). On the other hand, the death of OPBP1 overexpressing plants was significantly less compared with the control lines. The damage on the secondary leaves was more severe in the control lines than the OPBP1 overexpressing plants, as indicated by the Fv/Fm values on day 4 after the treatment. New emergent leaves in the Ubi:OPBP1 plants survived. The results suggest that overexpressing $O P B P 1$ increase the detoxification capability of rice.

Figure 4. The recovery growth of rice plant after salt stress.

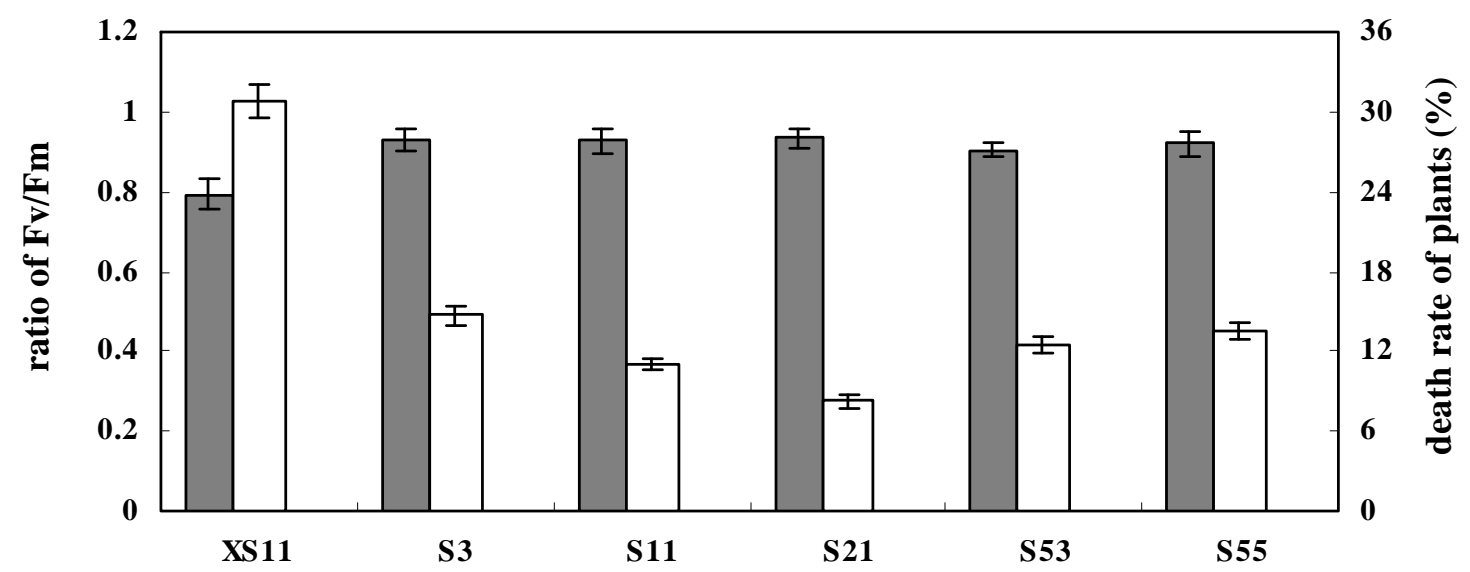

The control plant (XS11) and transgenic plants were treated with $250 \mathrm{mM} \mathrm{NaCl}$ solution for $18 \mathrm{~h}$, then they were allowed to grow under normal condition for recovery. The values of Fv/Fm were measured as described in Figure 3 and used as the ratio of day 4 to day one (the black column). The open column is the percentage of death plants.

2.3. Overexpression of OPBP1 increases tolerance to oxidative damage and resistance against rice fungal pathogens

Since generation of reactive oxygen species and programmed cell death are mediated in salinity stress [17], the tolerance against oxidative stress of the $O P B P 1$ overexpressing and control plants was studied by treatment the plants with paraquat $(\mathrm{Pq})$, which is a chemical producing superoxide anion and $\mathrm{H}_{2} \mathrm{O}_{2}$. Similarly, the $\mathrm{Fv} / \mathrm{Fm}$ values were measured to evaluate the damage of the plants. As shown in Figure 5, decreases of $\mathrm{Fv} / \mathrm{Fm}$ values were apparent in both the $O P B P 1$ overexpressing and the control plants $24 \mathrm{~h}$ after treatment with Pq. However, the OPBP1 overexpressing plants showed less 
extent of decrease in $\mathrm{Fv} / \mathrm{Fm}$ values comparing with the control plants, suggesting that overexpression of $O P B P 1$ stimulated the protective mechanism of rice plants against oxidative damage.

Figure 5. The effect of paraquat treatment on transgenic rice.

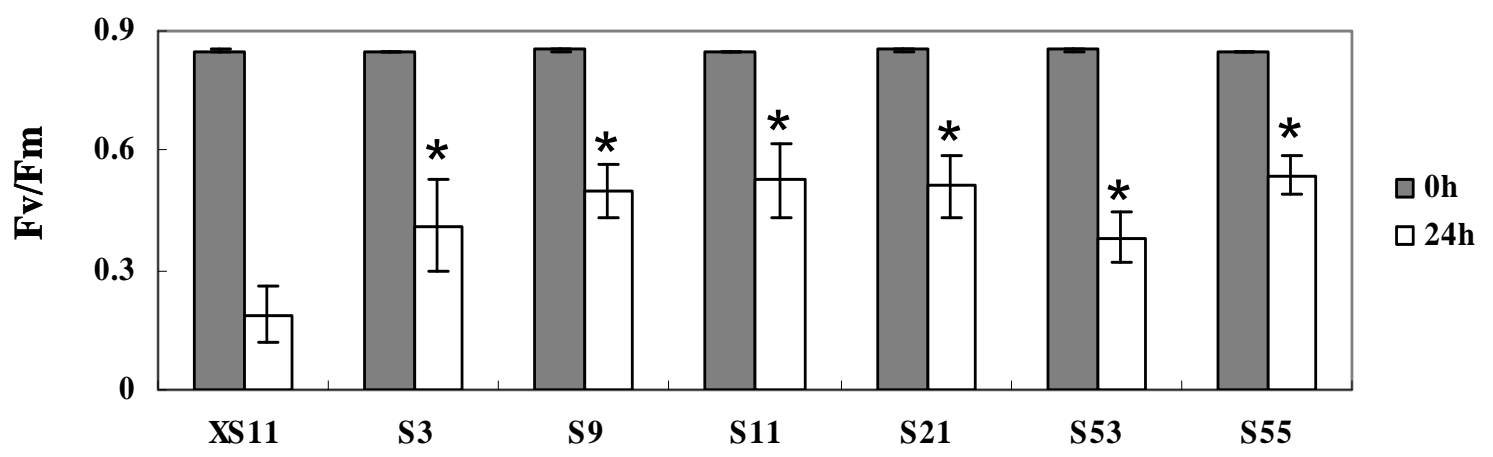

The control plant (XS11) and transgenic plants were treated with $30 \mu \mathrm{M}$ paraquat. $\mathrm{Fv} / \mathrm{Fm}$ values were measured $24 \mathrm{~h}$ after the treatment. Values are means of 10 different pieces, and bars are standard errors. Numbers indicate independent lines of transgenic $T_{2}$ plants. The values differ significantly $(\mathrm{P}<0.01)$ according to Fisher's protected LSD means comparison procedures.

Rice blast, caused by Magnaporthe oryzae, is a disastrous crop disease worldwide. To examine the effect of the transgene on disease resistance, the transgenic and control plants were inoculated with a virulent isolate of M. oryzae 97-220E3. Severity of disease development was evaluated by counting the number of lesions and measuring the size of lesions on infected leaves. The lesion numbers were reduced in the $O P B P 1$ overexpressing lines, especially lines 3, 11, and 21, comparing with the control plants (Figure 6A and 6B). Also, the lesions on the leaves of the transgenic plants were considerably smaller in size (Figure 6B).

Rice sheath blight is also a severe disease caused by Rhizoctonia solani. We inoculated the transgenic and control plants with an isolate of $R$. solani YN-7 and found that the lesion development was slower in the $O P B P 1$ overexpressing lines in comaprison with the control plants (Figure 6C).

\section{Discussion}

Genes induced by a stress are considered to play roles in responses to it. The early induced genes are required for stress perception, signal transduction or immediate protection, whereas those induced later are possibly involved in homeostasis and recovery. Overexpression of $O P B P 1$, an early immediate gene, results in a high tolerance to salt stress and enhanced resistance against pathogen attacks in tobacco [12]. Also, several reports have demonstrated that overexpression of ERF genes enhances the ability of transgenic plants to withstand environmental cues. Ectopic expression of CBF1/DREB1 genes activated cold responsive genes and enhanced cold and osmotic tolerance of non-acclimated Arabidopsis plants. DREB2, on the other hand, was found to be involved in drought-responsive gene expression [9, 10]. In this study, ectopic expression of OPBP1 gene enhanced tolerance to salt stress as well as resistance against $M$. oryzae and $R$. solani pathogens, suggesting OPBP1 probably functions in a similar way in rice as in tobacco. 
Figure 6. The resistance to Magnaporthe oryzae and Rhizoctonia solani of overexpression rice lines.

(A)

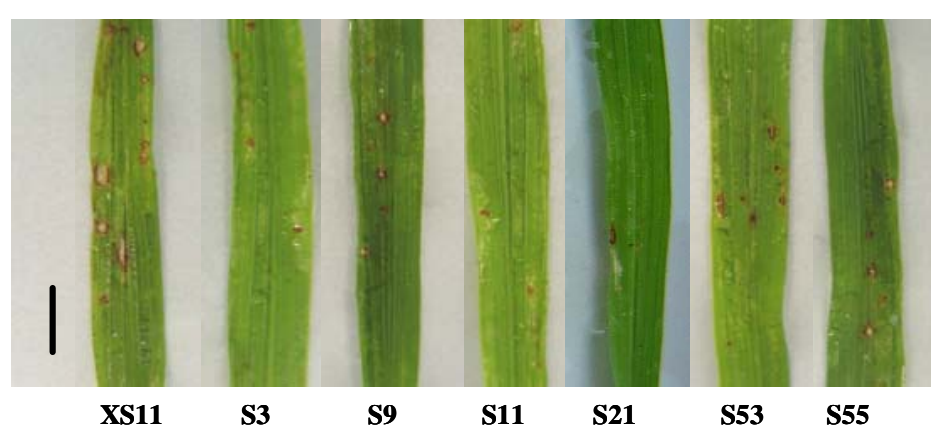

(B)

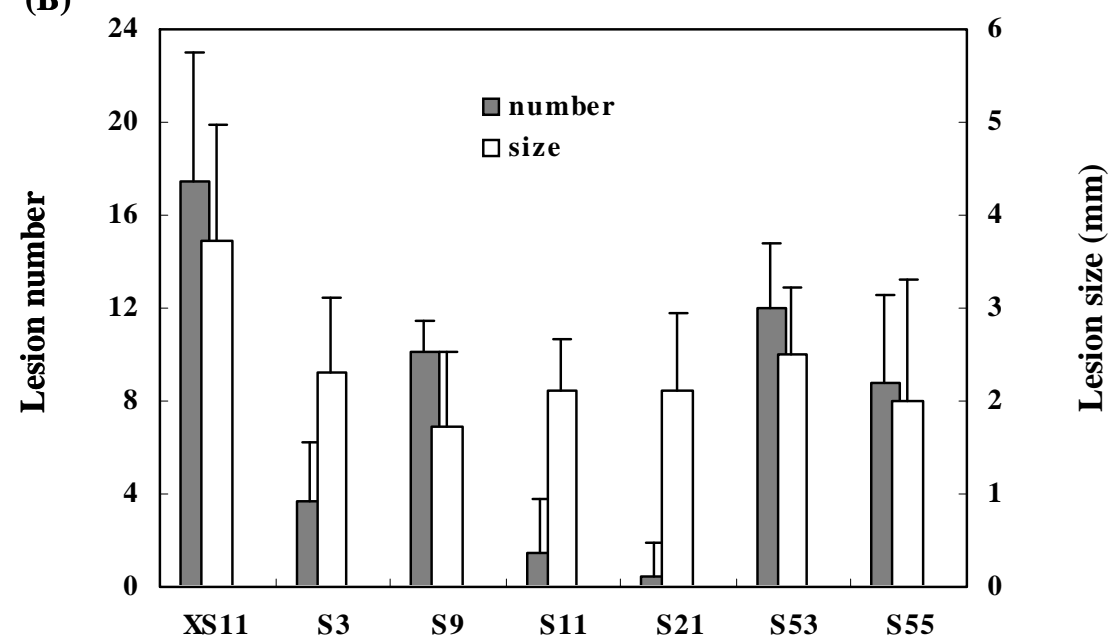

(C)

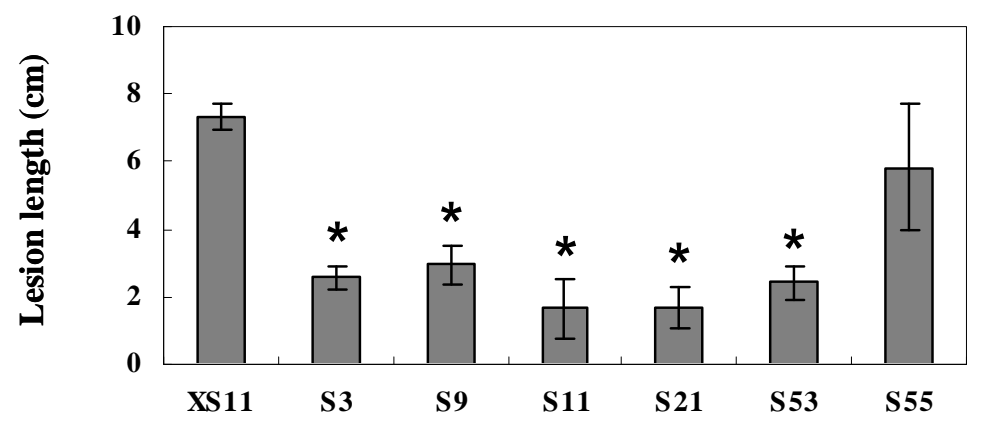

(A) The phenotype after inoculated with M. oryzae 97-220E3 for 7 days. The bar indicates $3 \mathrm{~mm}$. (B) Average lesion number and average lesion size $(\mathrm{mm})$. Values are means of 20 different plants detected in each transgenic line, and bars are standard errors. The values differ significantly $(\mathrm{P}<0.05)$ according to Fisher's protected LSD means comparison procedures. (C) Average lesion development length $(\mathrm{cm}) 13$ days post inoculation. Values are means of 10 different plants detected in each transgenic line, and bars are standard errors. The values of the overexpressing lines except S55 differ significantly $(\mathrm{P}<0.05)$ according to Fisher's protected LSD means comparison procedures. 
The damage cause by salt stress is often related to the production of oxidative stress. A number of reports have demonstrated that enhancement of the ROS scavenging systems in plants can provide protection from oxidative damage. For instance, overexpression of GST in tobacco showed substantial improvement of seedling growth under stressful condition including chilling and salinity [18]. This protective effect appears to scavenge products of lipid peroxidation generated as a result of enhanced ROS production. Overexpression of OPBP1 gene increased the level of OsGSTu expression except of line S7 (Figure 1B). This low OsGSTu expression level in line S7 seems to coincide with its lesser restoration capability from salt-stress (Figure 3A, 3D). Furthermore, OsIM gene is induced by salt stress and has been estimated to decrease the content of ROS generated under salt-stress condition [15]. OsIM shares homology with AtIM from Arabidopsis and PTOX from tomato, which function as a plastid terminal oxidase for carotenoid synthesis in the phytogene de-saturation step. Carotenoids can prevent plants from oxidative stress by reacting with reactive oxygen species directly or indirectly [19]. Increase the level of OsIM1 expression in $O P B P 1$ transgenic plants suggests again that the transgenic provides higher level of ROS scavenging capacity. In support of this, we observed a relative increase of paraquat tolerance in $O P B P 1$ overexpressing plants comparing with the controls (Figure 5).

One of the mechanisms for plant adaptation to water deficiency is the accumulation of low-molecular-weight organic compatible solutes such as sugars, some amino acids and quaternary ammonium compounds [20]. We examined the expression of some stress-related genes, such as OsP5CS, encoding a key enzyme for proline biosynthesis [13]. OsP5CS is induced by salt stress, cold stress, drought and the treatment of exogenous ABA [13]. The expression of OsP5CS and the accumulation of proline induced by high-salt treatment have been demonstrated to relate to the degree of salt tolerance in rice. In agreement, the increase in mRNA accumulation of OsP5CS in the transgenic plants suggest that overexpression of $O P B P 1$ may help avoid stress associated injuries and better recovery from stress. High salt stress disrupts homeostasis in water potential and ion distribution. To achieve salt tolerance, one of strategies is to help plants to re-establish both ionic and osmotic homeostasis in stressful environments. Increase osmotic stress by treatment with PEG increased root growths of transgenic and wild-type rice plants (Figure 3C), especially the increase in line S3 was significant different from the control plant coincident with the expression of OPBP1 in line S3, which was the highest among the plants studied. The results suggest that the high level of expressing OPBP1 in the transgenic plant probably lead more rapidly or strongly responsing to osmotic stress. The identification of molecular switches and regulatory genes would provide a better tool for crop improvement strategies.

\section{Experimental Section}

\subsection{Construction of plasmids and rice transformation}

To overexpress OPBP1 gene in rice (Oryza sativa subsp. japanica var. Xiushui 11), the cDNA of $O P B P 1$ was digested with BamH I/Sac I and inserted into pCoU linearized with the same enzymes. $O P B P 1$ gene was under the control of a maize ubiquitin promoter and terminated with an rbcs terminator in the generated plasmid (CoU-Ubi:OPBP1). Rice transformation was performed as described by Hiei et al. [21]. Hygromycin B-resistant plants were grown in the greenhouse and set seeds. 


\subsection{RNA isolation and northern blot}

Total RNA from rice leaves was isolated using the Trizol Reagent Kit according to manufacturer's instructions. An aliquot of $20 \mu \mathrm{g}$ total RNA was fractionated in a $1.0 \%$ denaturing agarose formaldehyde gel and subsequently transferred onto nylon membranes (Hybond-N+, Amersham). Hybridizations were performed with ${ }^{32} \mathrm{P}$-labeled $O P B P 1$ cDNA fragment as described previously [12].

\subsection{Chemical treatments}

The rice seeds of $\mathrm{T}_{2}$ progenies were surface sterilized with $70 \%$ ethanol for $5 \mathrm{~min}$ and with $2.5 \%$ $\mathrm{NaClO}$ for $30 \mathrm{~min}$. They were washed thoroughly, soaked in distilled water for $48 \mathrm{~h}$, and incubated for $24 \mathrm{~h}$ at $30{ }^{\circ} \mathrm{C}$ in the dark conditions. The germinated seeds were sown in $300 \mathrm{~mL}$ plastic cups with dark cloth outside. The seedlings were grown on the medium containing 1/2 MS salts without sucrose and organic ingredients, in the presence of $0,100,150$, and $200 \mathrm{mM} \mathrm{NaCl}$, respectively. For osmotic treatment, we used a $12.1 \%$ PEG6000 solution, which had a similar osmotic potential (-0.58 Mpa) as $100 \mathrm{mM} \mathrm{NaCl}$ solution. After 14 days growth at $25{ }^{\circ} \mathrm{C}$ with a day/night cycles of $16 / 8 \mathrm{~h}$, the heights and root length of the seedlings were measured.

To examine the recovery capability, the seedlings grew in $200 \mathrm{mM} \mathrm{NaCl}$ for two weeks were transfered to a new 1/2 MS medium in the absence of $\mathrm{NaCl}$ for 14 days more growth. Then, the seedlings were harvested for measurement of parameters. In order to investigate the seedlings tolerance to salt shock, the germinated seeds of the $T_{2}$ and the control lines were sown in pots filled with a mixture of soil and vermiculite $(1: 1, \mathrm{v} / \mathrm{v})$ and grown in the field under a 14/10 $\mathrm{h}$ (light/dark) photoperiod at $27{ }^{\circ} \mathrm{C}$ for 3 weeks with daily watering. The seedlings were treated or not with $250 \mathrm{mM}$ $\mathrm{NaCl}$ for $18 \mathrm{~h}$. After this treatment, the seedlings were washed with water and allowed to grow under normal condition for another 35 days. The values of $\mathrm{Fv} / \mathrm{Fm}$ of the secondary leaves were determined at the planned time. The dry weights were measured after placing fresh seedlings in an $80{ }^{\circ} \mathrm{C}$ oven for 72 hours.

For paraquat treatment, leaf blade pieces about $3 \mathrm{~cm}$ in length each from the secondary leaves of 3-week-old seedlings were floated on a $30 \mu \mathrm{M}$ paraquat solution. Equivalent leaf blade sections were incubated on sterile water without paraquat treatments as a control. The leaf blade sections were incubated for $24 \mathrm{~h}$ at $25{ }^{\circ} \mathrm{C}$ under continuous illumination $\left(200 \mathrm{~W} / \mathrm{m}^{2} / \mathrm{s}\right)$ until sampling.

\subsection{Determination of chlorophyll fluorescence}

Measurement of chlorophyll fluorescence was performed with a fluorometer. Fluorescence signals were from the second leaf of each rice plant, which had been dark-adapted for $15 \mathrm{~min}$, were measured at the indicated times. The ratio of $\mathrm{Fv}$ to $\mathrm{Fm}(\mathrm{Fv} / \mathrm{Fm})$ representing the activity of photosystem II was used to assess the functional damage to the plants [22].

\subsection{Pathogen inoculation}

A virulent (97-220E3) isolate of the blast fungus Magnaporthe oryzae was used in this study. Three-week-old seedlings of control and OPBP1 lines were inoculated with 97-220E3 at a concentration of $3 \times 10^{5}$ spores per ml, following the procedure described by Sallaud et al. [23]. After 
incubation in a dark chamber (at $22{ }^{\circ} \mathrm{C}$ and $95 \%$ relative humidity) for $24 \mathrm{~h}$, plants were moved to a growth chamber and maintained at $28^{\circ} \mathrm{C}$ with $16 \mathrm{~h}$ of light and $8 \mathrm{~h}$ of dark cycles. Disease symptoms were evaluated and photographed. Number of lesions per leaf was counted as the size of the lesions was measured. The experiment was repeated three times in rice seedlings with similar results.

The isolate of Rhizoctonia solani (YN-7) was used in this study. Three-week-old seedlings of control and OPBP1 lines were inoculated with YN-7, following the procedure described by Jia et al. [24]. The greenhouse temperate averaged $28{ }^{\circ} \mathrm{C}$, and relative humidity was maintained within $70 \%$ to $80 \%$. Lesion development was monitored starting 3 days post inoculation. The length of the lesion was measured. Sheath blight micro-chamber inoculations were repeated twice.

\subsection{RT-PCR analysis}

To remove possible DNA contamination, the total RNA was treated with DNase, then was heated at $70{ }^{\circ} \mathrm{C}$ for $10 \mathrm{~min}$ to inactivate the DNase, then followed by phenol-chloroform extraction and ethanol precipitation. The first strand cDNAs were synthesized by using the AMV reverse transcriptase system in $20 \mu \mathrm{L}$ reactions, including $1 \mu \mathrm{g}$ purified total RNA. After synthesis, the cDNA was diluted and used as template for PCR amplifications in $50 \mu \mathrm{L}$ standard reactions. For RT-PCR, actin was used as internal constitutive control. The PCR products were separated on $1.6 \% \mathrm{w} / \mathrm{v}$ agarose gels, stained with ethidium bromide, and photographic images were obtained. The primers used in the study were as followings: rice actin (GenBank accession number: AB047313), OsACTIN-F: 5'-ggaactggtatggtcaaggc-3' and OsACTIN-R: 5'-agtctcatggatacccgcag-3'; OPBP1 (GenBank accession number: U81157), OBP1BI: 5'-acgaggaaaaacaaaatggattct-3' and OBP1R: 5'-atggaaacaaagagatggaattccctat-3'; OsP5CS (GenBank accession number: D49714), OsP5CS-F: 5'-tggatgtctcgtcatctcaact-3' and OsP5CS-R: 5'-aagccaagacagcagccttcac-3'; OsGSTu1 (GenBank accession number: AF050102), OsGSTu1-F: 5'-aagctatagccatggcggaggag-3' and OsGSTu1-R: 5'-tgcaacttcgcatccaccgatctac-3'; OsIM1 (GenBank accession number: AF085174), OsIM1-F: 5'-gggagaaggagcagaccga-3' and OsIM1-R: 5'-gccacccaattcttccatg-3'. Transcript abundance of OsActin in leaves was 25cycles of PCR amplification.

\section{Conclusions}

$O P B P 1$ gene, an AP2/ERF transcription factor of tobacco, seems to be an important determinant of biotic and abiotic stress response in plants. Transgenic rice plants with overexpressed OPBP1 gene accumulated higher levels of OsP5CS, OsGSTu1 and OsIM1, which are reported to be involved in the signaling pathways of salt or oxidative stress tolerant. OPBP1 overexpression lines exhibited enhanced tolerance to salt and oxidative stress, as well as to infection of Magnaporthe oryzae and Rhizoctonia solani pathogens. These results suggest that expression of OPBP1 gene increase the detoxification capability of rice.

\section{Acknowledgements}

This work was supported by Commonweal Specialized Research Fund of China Agriculture (3-16) and Research Fund for the Doctoral Program of Higher Education of China (20070019066). 


\section{References}

1. Ramanjulu, S.; Bartels, D. Drought- and desiccation-induced modulation of genes expression in plants. Plan Cell Environ. 2002, 25, 141-151.

2. Wise, M.; Tunnaciliffe, A. POPP the question: What do LEA proteins do? Trends Plant Sci. 2004, 9, 13-17.

3. Garg, A.K.; Kim, J.K.; Owen, T.G.; Ranwala, A.P.; Choi, Y.D.; Kochian, L.V.; Wu, R.J. Trehalose accumulation in rice plants confers high tolerance levels to different abiotic stresses. Proc. Natl. Acad. Sci. USA 2002, 99, 15898-15903.

4. Ohme-Takagi, M.; Shinshi, H. Ethylene-inducible DNA binding proteins that interact with an ethylene-responsive element. Plant Cell 1995, 7, 173-182.

5. Liu, Q.; Kasuga, M.; Sakuma, Y.; Abe, H.; Mirua, S.; Yamaquchi-Shinozaki, K.; Shinozaki, K. Two transcription factors, DREB1 and DREB2, with an EREBP/AP2 DNA-binding domain separate two cellular signal transduction pathways in drought and low-temperature-responsive gene expression in Arbidopsis. Plant Cell 1998, 10, 1391-1406.

6. Nakano, T.; Suzuki, K.; Fujimura, T.; Shinshi, H. Genome-Wide analysis of the ERF gene family in arabidopsis and rice. Plant Physiol. 2006, 140, 411-432.

7. Tang, W.; Newton, R.J.; Lin, J.; Charles, T.M. Expression of a transcription factor from Capsicum annuum in pine calli counteracts the inhibitory effects of salt stress on adventitious shoot formation. Mol. Genet. Genomics 2006, 276, 242-253.

8. Jung, J.; Won, S.Y.; Suh, S.C.; Kim, H., Wing, R.; Jeong, Y., Hwang, I., Kim, M. The barley ERF-type transcription factor HvRAF confers enhanced pathogen resistance and salt tolerance in Arabidopsis. Planta 2007, 225, 575-588

9. Stockinger, E.J.; Gilmour, S.J.; Thomashow, M.F. Arabidopsis thaliana CBF1 encodes an AP2 domain-containing transcription activator that binds to the C-repeat/DRE, a cis-acting DNA regulatory element that stimulates transcription in response to low temperature and water deficit. Proc. Natl. Acad. Sci. USA 1997, 94, 1035-1040

10. Kasuga, Ml; Liu, Q.; Miura, S.; Yamaguchi-Shinozaki, K.; Shinozaki, K. Improving plant drought, salt, and freezing tolerance by gene transfer of a single stress-inducible transcription factor. Nat. Biotech. 1999, 17, 287-291

11. Dubouzet, J.G.; Sakuma, Y.; Ito, Y.; Kasuga, M., Dubouzet, E.G.; Miura, S.; Seki, M.; Shinozaki, $\mathrm{K}$. OsDREB genes in rice, Oryza sativa L., encode transcription activators that function in drought-, high-salt- and cold-responsive gene expression. Plant J. 2003, 33, 751-763.

12. Guo, Z.J.; Chen, X.J.; Wu, X.L.; Ling, JQ.; Xu, P. Overexpression of the Ap2/EREBP transcription factor OPBP1 enhances disease resistance salt tolerance in tobacco. Plant Mol. Biol. 2004, 55, 607-618.

13. Igarashi, Y.; Yoshiba, Y.; Sanada, Y.; Yamaguchi-Shinozaki, K.; Wada, K.; Shinozaki, K. Characterization of the gene for $\triangle 1$-pyrroline-5-carboxylate synthetase and correlation between the expression of the gene and salt tolerance in Oryza sativa L. Plant Mol. Biol. 1997, 33, $857-865$. 
14. Soranzo, N.; Sari-Gorla, M.; Mizzi, L.; De-Toma, G.; Frova, C. Organisation and structural evolution of the rice glutathione S-transferase gene family. Mol. Gen. Genomics 2004, 271, $511-521$.

15. Kong, J.; Gong, J.M.; Zhang, Z.G.; Zhang, J.S.; Chen S.Y. A new AOX homologous gene OsIM1 from rice (Oryza Sativa L.) with an alternative splicing mechanism under salt stress. Theor. Appl. Genet. 2003, 107, 326-331.

16. Zhu, J.K. Plant salt tolerance. Trends Plant Sci. 2001, 6, 66-71.

17. Hasegawa, P.M.; Bressan, R.A.; Zhu, J.K.; Bohnert H.J. Plant cellular and molecular response to high salinity. Annu. Rev. Plant Physiol. Plant Mol. Biol. 2000, 51, 463-499.

18. Roxas, V.P.; Smith, R.K.Jr.; Allen, E.R.; Allen, R.D. Overexpression of glutathione-S-transferase/glutathione peroxidase enhances the growth of transgenic tobacco seedlings during stress. Nat. Biotechnol. 1997, 15, 988-991.

19. Demmig-Adams, B.; Gilmore, A.M.; Adams Ш, W.W. Carotenoids 3: in vivo functions of carotenoids in higher plants. FASEB J. 1996, 10, 403-412.

20. Bohnert, H.J.; Nelson, D.E.; Jensen, R.G. Adaptations to Environmental Stresses. Plant Cell 1995, 7, 1099-1111.

21. Hiei, Y.; Ohta, S.; Komari, T.; Kumashiro, T. Efficient transformation of rice (Oryza sativa L.) mediated by Agrobacterium and sequence analysis of the boundaries of the T-DNA. Plant J. 1994, 6, 271-282.

22. Moradi, F.; Ismail, A.M. Responses of Photosynthesis, Chlorophyll Fluorescence and ROS-Scavenging Systems to Salt Stress During Seedling and Reproductive Stages in Rice. Ann. Bot. 2007, 99, 1161-1173.

23. Sallaud, C.; Lorieux, M.; Roumen, E.; Tharreau, D.; Berruyer, R.; Svestasrani, P.; Garsmeur, O.; Ghesquiere, A.; Notteghem, J.L. Identification of five new blast resistance genes in the highly blast-resistant rice variety IR64 using a QTL mapping strategy. Theor. Appl. Genet. 2003, 106, 794-803.

24. Jia, Y.; Correa-Victoria, F.; McClung, A.; Zhu, L.; Liu, G., Wamishe, Y. Rapid Determination of Rice Cultivar Responses to the Sheath Blight Pathogen Rhizoctonia Solani Using a Micro-Chamber Screening Method. Plant Disease 2007, 91, 485-489.

(C) 2008 by the authors; licensee Molecular Diversity Preservation International, Basel, Switzerland. This article is an open-access article distributed under the terms and conditions of the Creative Commons Attribution license (http://creativecommons.org/licenses/by/3.0/). 